\title{
The August 11th, 1999 CME
}

\author{
S. Koutchmy ${ }^{1}$, F. Baudin ${ }^{2}$, K. Bocchialini ${ }^{2}$, J.-Y. Daniel ${ }^{1}$, J.-P. Delaboudinière ${ }^{2}$, L. Golub ${ }^{3}$, \\ P. Lamy ${ }^{4}$, and A. Adjabshirizadeh ${ }^{5}$
}

\author{
1 Institut d'Astrophysique de Paris, CNRS, 98 bis Boulevard Arago, 75014 Paris, France \\ 2 Institut d'Astrophysique Spatiale, Université Paris XI/CNRS, Bâtiment 121, 91405 Orsay Cedex, France \\ ${ }^{3}$ Smithsonian Astrophysical Observatory/Center for Astrophysics, Cambridge, Massachussets, USA \\ ${ }^{4}$ Laboratoire d'Astronomie de Marseille, CNRS, BP 8, 13376 Marseille, France \\ 5 Department of Astronomy, University of Tabriz, Tabriz, Iran
}

Received 15 August 2001 / Accepted 2 February 2004

\begin{abstract}
We present here a set of observations, space borne and ground based, at different wavelengths, of the solar corona at and after the time of the total solar eclipse of August 11th. It is used to consider some unusual features of the coronal dynamics related to a limb Coronal Mass Ejection (CME) observed after the total eclipse. The complementary aspect of simultaneous ground-based and space-borne observations of the corona is used to produce an accurate composite image of the White Light (W-L) corona before the CME. A high arch system (possibly a dome-like structure, with large cavities inside but without a cusp further out) which appeared on the eclipse W-L images, is suggested to be a large-scale precursor of the CME, well preceding the eruption of the top part of the brightest prominence recorded in W-L. This bright prominence is shown as a filament in absorption using the Transition Region And Corona Explorer (TRACE) images taken in different coronal lines. The analysis of the images of the Large Angle and Spectrometric Coronograph (LASCO) on board the Solar and Heliospheric Observatory (SoHO), showing the progression of the CME, is discussed in an attempt to make a connection with the surface event. A SoHO-EIT (Extreme UV Imager Telescope) image sequence details the prominence eruption and shows the sudden heating processes of the ejected parts. We found that there is no reason to assume that the huge cavity is significantly destabilised well before the eruption of the upper part of the low-lying bright twisted filament which coincides with the position of one of the legs of the high arch. Observations are still compatible with the assumption of both the break-out model and of the flux rope erupting model as a result of a shear or of an increasing poloïdal magnetic flux from below. We stress the possible role of buoyancy of the giant cavity as a destabilizing factor leading to the CME, noticing that some motion of coronal material back toward the surface can be seen during at least the first phase of the CME, from both EIT and LASCO observations.
\end{abstract}

Key words. Sun: corona - Sun: coronal mass ejections (CMEs)

\section{Introduction}

The analysis of dynamical phenomena occurring inside the solar corona is of fundamental importance to understand the basic physical processes leading to the high temperature of the coronal plasma and, ultimately, to understand the mechanism(s) of mass loss of the solar atmosphere. Total solar eclipses are excellent opportunities to perform measurements on both the white-light (W-L) corona and the spectrum of the corona, due to the large flux of photons provided by the visible corona. Three superposed components of the corona are observed during an eclipse:

- the W-L structured corona (K), due to the Thomson scattering on free electrons;

- the emission lines (E) from the 1 to 3 millions Kelvin ions;

- the more diffuse component $(\mathrm{F})$ due to the forward scattering by small dust grains orbiting the Sun.

Send offprint requests to: S. Koutchmy, e-mail: koutchmy@iap.fr
During a total eclipse, although just a snapshot is usually possible, the occultation produced by the Moon is ideally suited to analyze the parts of the corona which are always hidden behind the external occulting system and in addition, by internal diaphragms, when a spaceborne coronagraph is used. This hidden part contains the main coronal mass, up to 0.8 of the mass of the whole K-corona. After subtracting the stationary contribution of the F-corona (Koutchmy \& Lamy 1985), the resulting W-L images (K-corona) reflect directly and linearly the distribution of electron densities in plasma structures, providing that the effect of the dilution factor, which decreases as the square of the radial distance, is removed. Coronal lines fluxes (E-corona) decrease in the inner corona more like the power 1.7 to 2 of the electron densities. Formulas to interpret the intensities integrated along the line of sight are well established and repeatedly published (see e.g. Koutchmy 1992 and 1994 for results regarding the hydrostatic non-isothermal case). To evaluate densities in W-L loops and arches, November \& Koutchmy (1996) gave practical and more precise formulae. It must be 
pointed out that the usual values of electron densities given in the literature are indeed strongly related to assumptions made to describe the coronal 3D structures, taking into account the spatial resolution of observations. However, the 3D structure of the corona is often ignored or neglected and standard values from Allen's revised book (2000) can be used as a reference.

Among dynamical phenomena well observed in the corona, coronal mass ejections (CMEs) are one of the most spectacular events occurring in the solar atmosphere, and, presumably, inside all extended and active stellar atmospheres. They are a direct manifestation of the emergence and evolution of the changing solar magnetic field. Their occurrence follows the 11.3 year cycle of activity. These events represent thousands of millions of tons of solar material ejected into the heliosphere in about $1 \mathrm{~h}$. The ejected particles have average speeds of several hundreds $\mathrm{km} \mathrm{s}^{-1}$ and may reach the Earth atmosphere after some days, disturbing the magnetosphere and sensitive electronic systems. When a big flare is released, even more energetic (sub-relativistic) particles reach the low Earth atmosphere with additional consequences. At time of maximum activity, CMEs occur quite often and the probability of observing such an event during an eclipse is high. The best documented case is the limb rather fast CME observed during the eclipse of Feb. 15, 1980, noticeable also because it showed some evidence of twisted "legs" (Airapetian \& Koutchmy 1994).

Many works and several conferences, both observational and theoretical, have been dedicated to the study of CMEs. However, the basic mechanism at the origin of the mass ejections is still unknown, although it is clear that, to some extent, the coronal magnetic field is the acting agent (see, among many other works, Luhmann et al. 2003). A mechanism considered by theoreticians (e.g. Gibson \& Low 1998) is based on a scenario implying the basic "dipolar" structure of an axisymmetric equatorial helmet streamer with a cusp above and a long stalk further out. Magnetic reconnections are assumed to destabilize the whole system and induce an eruption of the inserted lower prominence. This CME model seems not applicable for i) the recent LASCO/SoHO observations which show a rather stable streamer-belt producing the helio-sheet during long periods of time at solar minimum, and for ii) those showing huge halo CMEs disturbing streamers during their passage, at solar maximum. From the observational point of view, overlapping effects along the line of sight and the masking effect produced by the external occulter of the coronagraph make the evaluation of the CME origins difficult inside a helmet streamer belt. However, this model contains the basic observed ingredients of CMEs: the arching, the cavity and the erupting prominence. A more complex configuration, quadrupolelike, was later considered by Antiochos et al. (1999), who proposed a break-out model. A rather different mechanism has been worked out by Chen in 1989 (see e.g. Krall et al. 2000; Chen et al. 2000), based on the detailed analysis of the dynamical properties of a large-scale erupting magnetic flux rope surrounded by a rather homogeneous but horizontal field. Figure 1 presents sketches to summarize the main properties of these two different approachs. We did not include models apparently producing a CME as the result of a large explosion at the solar surface like a flare. This last class of events is better analyzed as
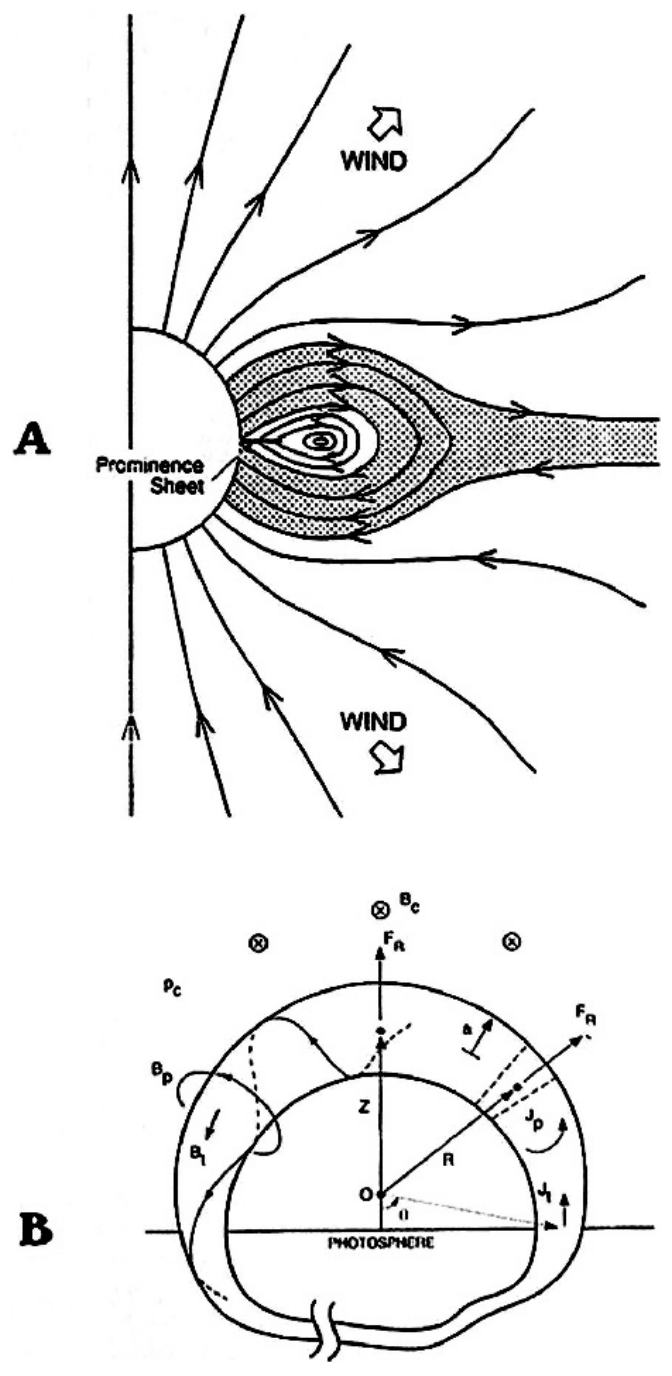

Fig. 1. Different models for CMEs. Model A: "Dipolar model" of Gibson \& Low (1998); Model B: erupting toroïdal flux rope of Chen et al. (2000).

X-ray jets or even W-L linear rays (Koutchmy \& Nikoghossian 2002).

In this contribution, we first present observations taken during the 1999 total eclipse in Iran which occurred a few hours before a large CME was observed from space. The region in which the CME originates shows a faint but unusual high arch structure, without helmet or stalks above. We suggest that it is a "precursor" of the CME. SoHO and TRACE observations are also considered to discuss additional more dynamical aspects of the event and to demonstrate the significance of the W-L eclipse data. This observation contributes new insights into the problem of filament activation and eruption when considering the coupling with the cavity bright edge dynamical phenomenon and the complex cavity structure inside. These topics are often the subject of debate in understanding the origin of CMEs (for a more complete presentation of the topic, see Harrison 2000). 


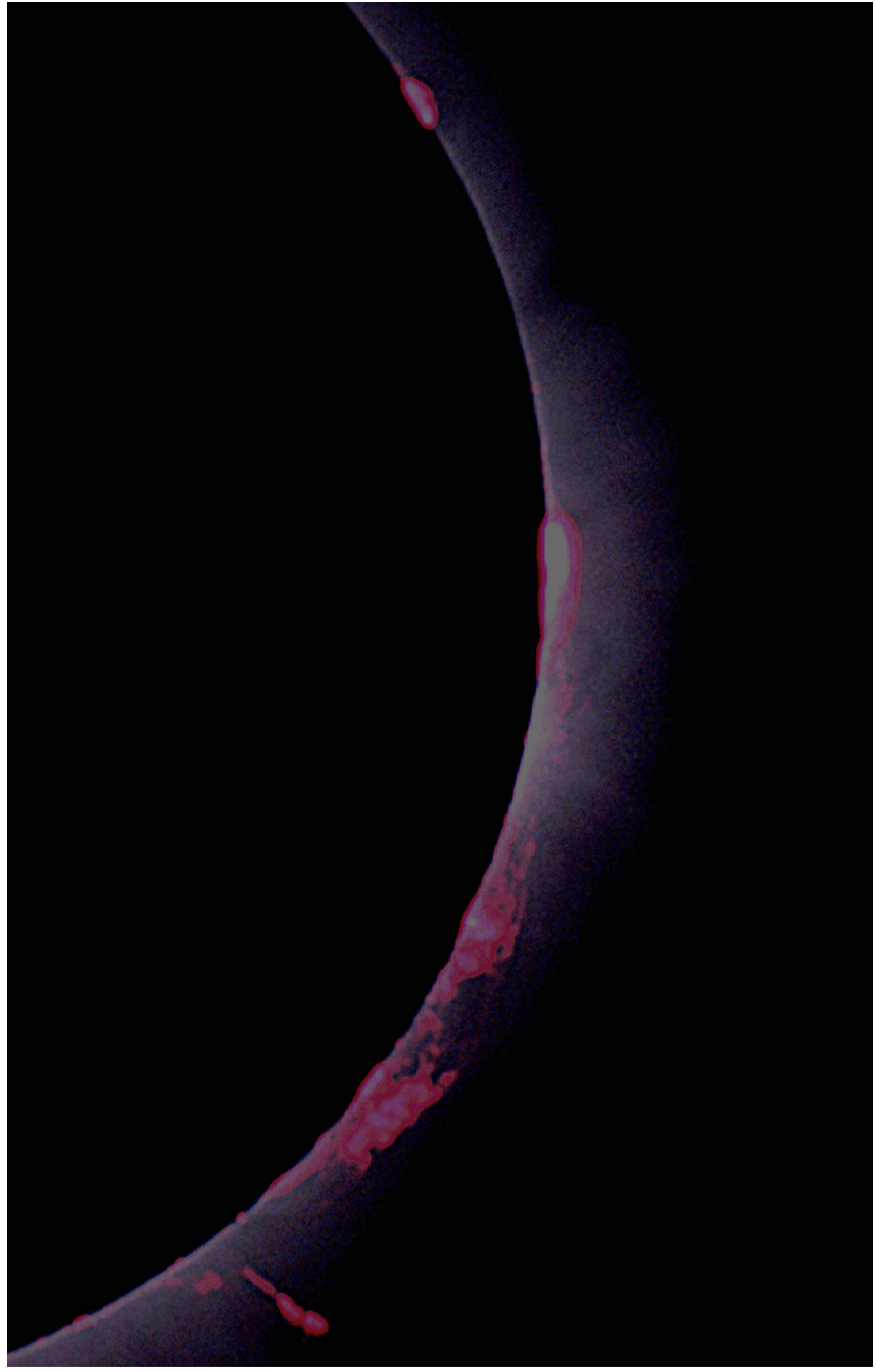

Fig. 2. Short exposure eclipse color image (partial frame) made in Iran at 12:03 UT (by M. Laal Aaly), showing the chromospheric structures on the West limb and the overlying coronal structures. The brightest prominence at West-North-West (WNW) will erupt several hours after the eclipse, as observed with EIT. Here and thereafter, North is up and East is to left.

\section{Observational material and results}

Some preliminary results have already been given in Adjabshirizadeh et al. (1999) together with a brief description of our observations. Our program included imaging (including a radial neutral filter), polarisation and spectroscopic observations. Only imaging results are used in this work. We successfully operated the experiments over a clear sky. Figure 2 shows the West limb of a typical short exposure colour image of the inner corona. Chromospheric structures are readily recognised by their dominant red/pink colour due to emissions of the "cool" $\mathrm{H} \alpha$ and $\mathrm{H} \beta$ lines and the He I D3 line. The brightest regions of the hot corona, especially at the NE and at WNW limbs of the full corona image (not shown in Fig. 2) are also noticed in W-L. This demonstrates that the density there is high.

To analyse the chromospheric structures inserted in the inner corona, especially the faint prominences, it is important to compare our observations with spaceborne EUV images.

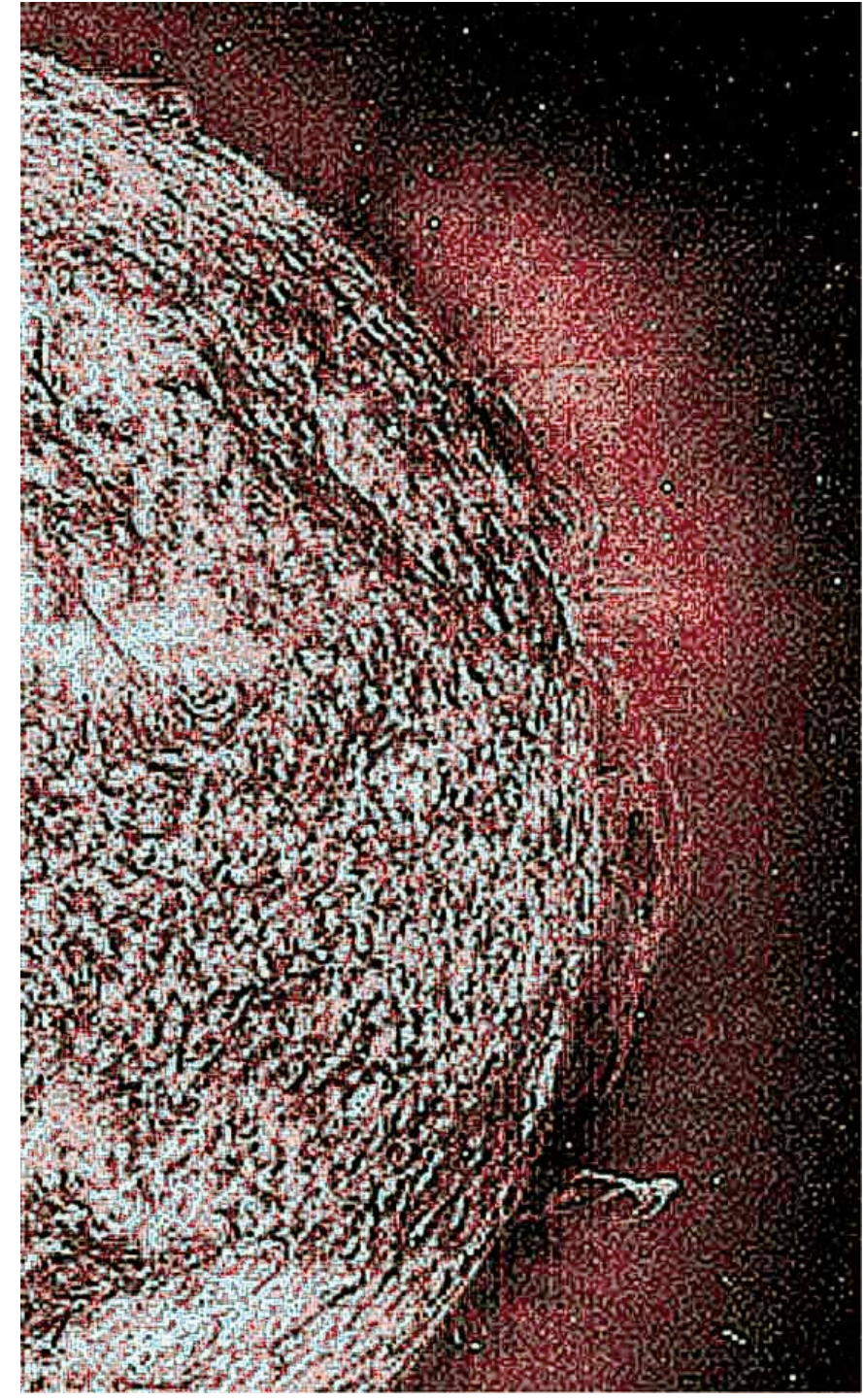

Fig. 3. The same frame as Fig. 2, but taken simultaneously in the $\mathrm{He}$ II channel at $30.4 \mathrm{~nm}$ with the EIT experiment of SoHO.

Because we are dealing with highly dynamical phenomena, it is interesting to compare images taken exactly at the same time. Images made with EIT on SoHO are the most appropriate since they are obtained with a short enough exposure time. Figure 3 shows the W limb part of the EIT image at $30.4 \mathrm{~nm}$ corresponding mainly to the resonance HeII line produced in regions where the temperature is of the order of $50000 \mathrm{~K}$. This simultaneous imaging was performed with a precision of a minute which is good enough, taking into account the spatial resolution of the images. Several faint prominences can readily be identified on both the eclipse and the EIT images. However, a more accurate examination shows that their relative intensities, and possibly their morphology, are quite different. The brightest prominence (which will erupt later on) visible in Fig. 2 is hard to see in Fig. 3 where it is indeed appearing in absorption. This seems to be related to the problem of different temperatures of the parts of prominences shown in different emission lines. 


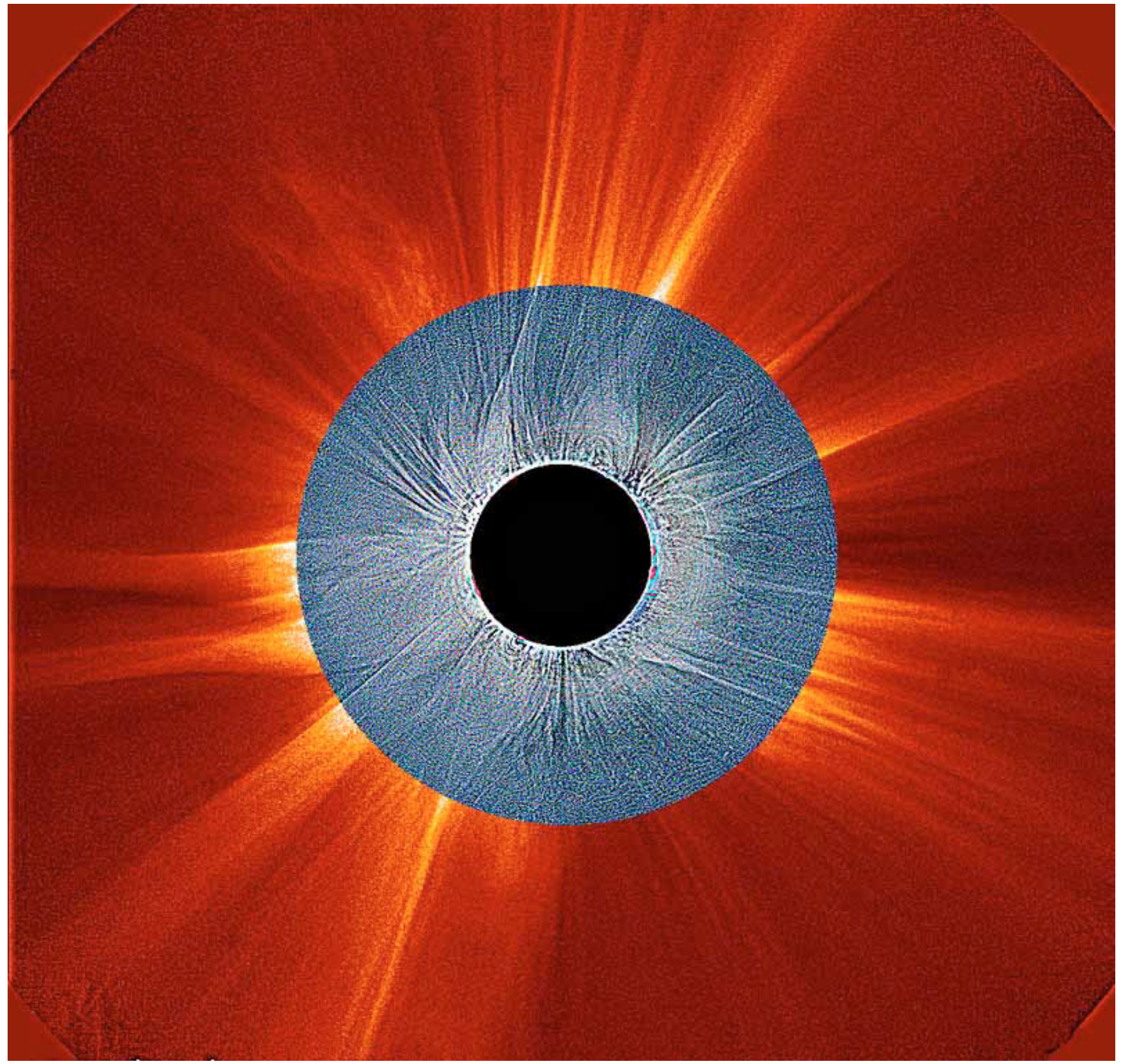

Fig. 4. Composite image of the corona at the time of the eclipse. The inner part is made of the W-L image obtained in Iran by J. Mouette at 12:03 UT with a radial gradient neutral filter. The outer part is made of the Lasco-C2 on SoHO image made at 12:06 UT Note the perfect correspondence of the radial fine structures, and the unusual high arch at WWN, without evidence of a cusp further out. A large part of the W limb seems to show only open rays. Edge enhancement processing has been applied to better show the structure of the corona, increasing the noise of the film of the eclipse image. (This figure is available in color in the electronic version.)

Considering the more extended W-L corona, we show the complementary nature of eclipse and spaceborne observations. Due to the use of their external occulters, spaceborne coronagraphs cannot properly study the inner corona, typically the region situated below 2 solar radii from the Sun center (about 2.5 solar radii with the Lasco-C2 coronagraph of SoHO). During the eclipse totality, this region is the brightest and is very easy to image, the limitation being given by the apparent diameter of the Moon which is never significantly larger than the solar diameter (their ratio defines the magnitude of the eclipse, equal to 1.025 in Iran).
Accordingly, we have a perfect case of complementary observations. Because the same object is observed from almost the same point of view, we should have a perfect agreement in radial directions when the continuity of the smallest structures is considered. This is indeed the case as illustrated in Fig. 4.

Beside the formal demonstration of the complementarity of ground-based and spaceborne observations, this composite image once again demonstrates that the distribution of electron densities inside a streamer can better be described assuming they are made of folded sheets presumably extending above the neutral magnetic lines where $\mathrm{B} \rho$ (the radial 


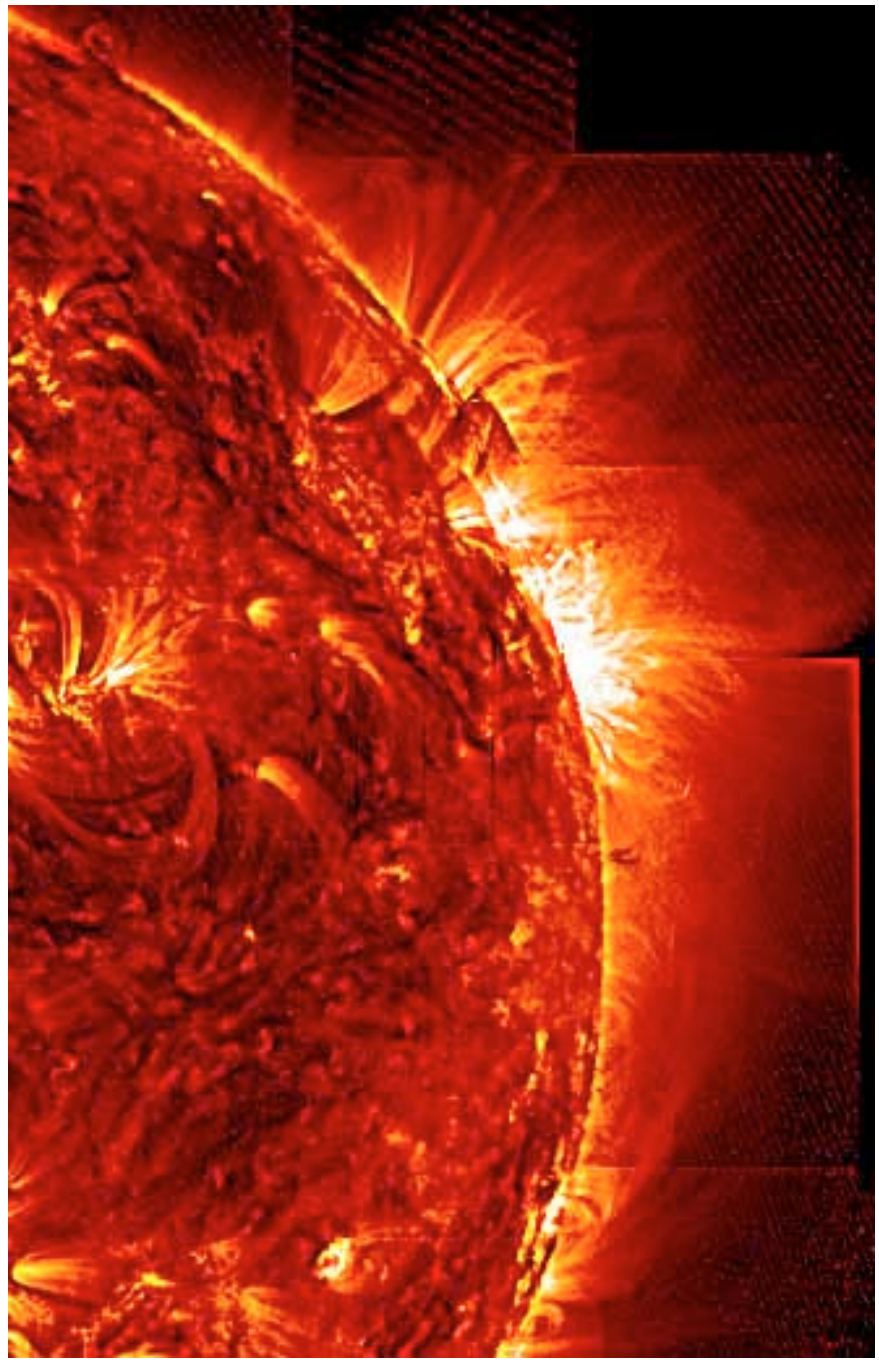

Fig. 5. Composite TRACE image showing the WNW region using the Fe IX channel at $17.1 \mathrm{~nm}$, typically $1 \mathrm{~h}$ after the totality in Iran. The WNW prominence can be seen in absorption, although emissions from the bright surrounding coronal enhancement partly masks it.

component of the magnetic field) is zero, as theoretically proposed by Molodensky et al. (1996). Finally, there are several arch-like structures seen around the main limb prominences, at ESE, NWN and SWS (see Adjabshirizadeh et al. 1999). The high arch at WNW is remarkable: it is made of a main arch which is definitely closed at the top. It reaches almost 3/4 solar radius above the surface which means many scale heights (the scale height changes from $50 \mathrm{Mm}$ in the very inner corona to $100 \mathrm{Mm}$ in the intermediate corona (see Koutchmy 1992, 1994; November \& Koutchmy 1996). In addition, no cusp is seen above (see Fig. 4) and the background corona looks open around the structure. Below this structure, we see a complex coronal cavity with an enhancement inside (Figs. 5, 6 and 10) and, closer to the surface, the very bright prominence which erupted several hours after the eclipse.

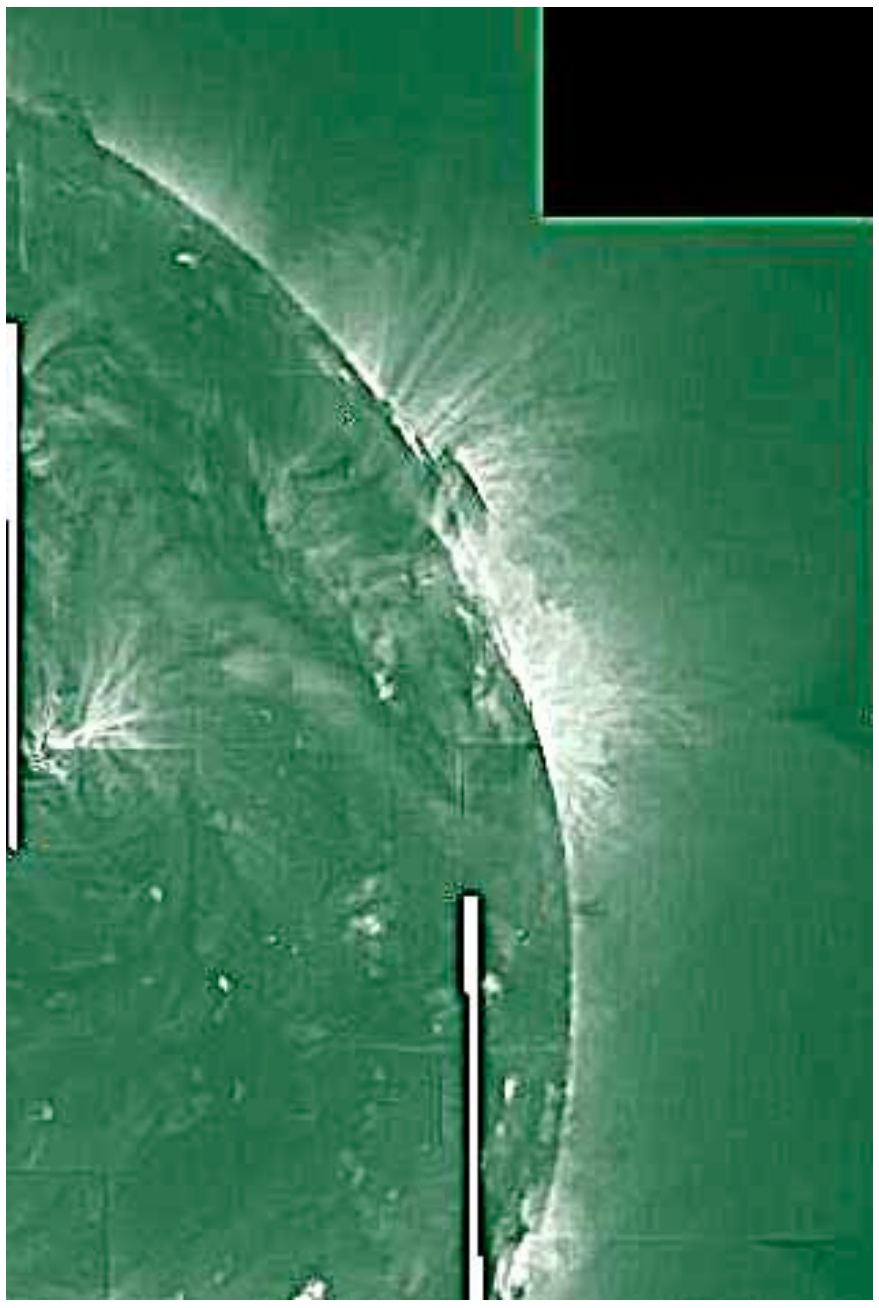

Fig. 6. The same field as Fig. 5 but taken in the TRACE $19.5 \mathrm{~nm}$ channel of Fe XII, $20 \mathrm{mn}$ later. Note the rather intense coronal enhancement nearby but lower than the dark prominence at WNW which will later partly erupts.

\section{The large dynamical event (CME) at WNW}

Approximately $9.5 \mathrm{~h}$ after the totality in Iran, a part of the bright low-lying prominence seen at WNW erupted and a classical large limb CME was recorded $2 \mathrm{~h}$ later on both Lasco-C2 and (later) C3 images.

\subsection{EIT observations}

The prominence ejection is well described by the routine full disk imaging (the "CME watch" program) performed with EIT in the $19.5 \mathrm{~nm}$ channel of FeXII every $12 \mathrm{mn}$ (Figs. 7 and 8). These figures were built using partial frames and selected times corresponding to the prominence system eruption and ejection. Note that only the upper part of the bright prominence erupted, with possibly signatures of non-radial twisting, by expanding and suddenly heating the whole set of filamentary structures to coronal temperatures. This is obvious because the same moving parts of the prominence are recognised first, as seen in absorption (cool plasma), and after in emission (hot plasma). This process of "dynamical heating" is two-fold: a hidden source of 


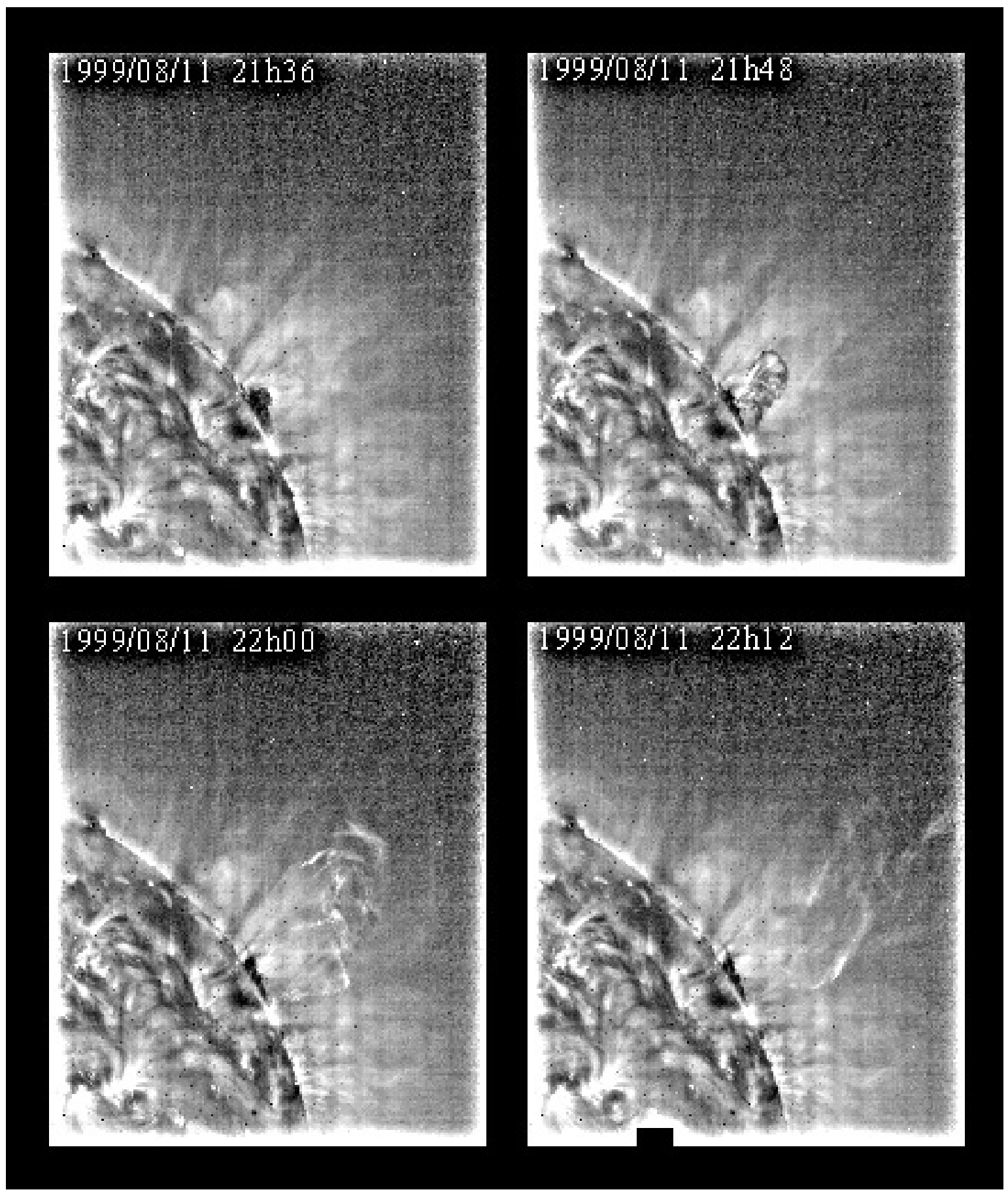

Fig. 7. Four partial frames from EIT images taken in the $19.5 \mathrm{~nm}$ channel of Fe XII reveal the eruption of the upper part of the WNW limb prominence, with "hot" (bright) and "cool" (dark) structures. There is an obvious but unidentified rapid extended heating processing during the motion of the prominence threads. Edge enhancement image processing has been applied to make the phenomenon clearer.

activation of the erupting filaments exists, producing the accelerated motions and slightly after, during the expansion, heating takes place. To solve the problem, theoreticians propose magnetic reconnections as a source of heating and of accelerating particles (e.g. Priest \& Forbes 2000) but here, this mechanism is difficult to test. First, we notice that the components of the ejected part of the prominence keep approximately their identity during the sudden heating, although the scale is greatly expanded and the whole system moves outward, working against gravity. Second, it is not clear where reconnections are taking place. The surrounding corona seems not to be involved or its effect is weak. It seems that the source of heating is inside each thread, as it would be for ropes heated by Joule dissipation of electric currents produced in the erupting prominence filaments as the result of a short-circuit. The discussion of the whole process implying both the coronal heating and the dynamics needs a careful analysis which is beyond the scope of this paper. However, a possible explanation of the heating 


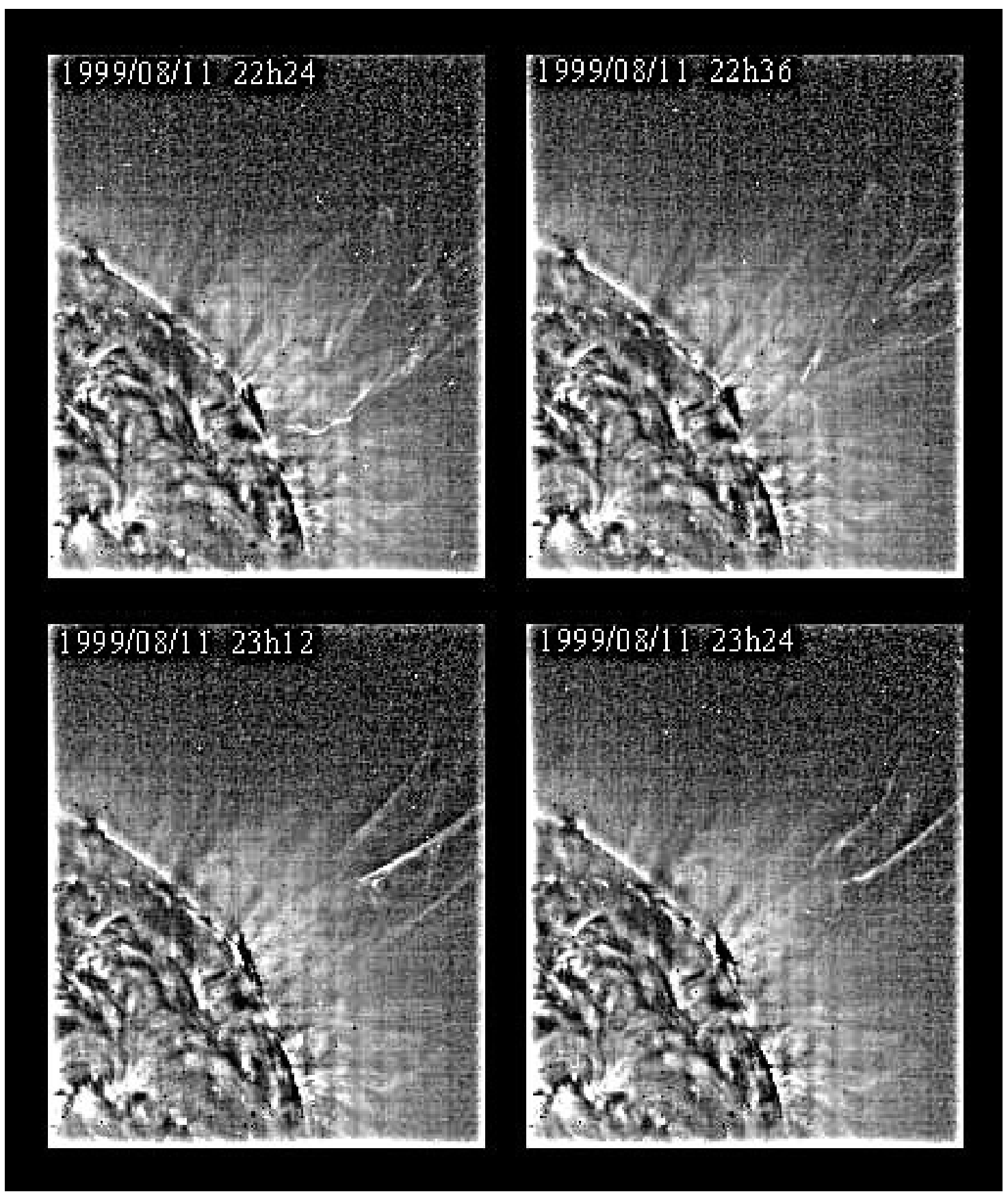

Fig. 8. As in Fig. 7 but later on. Note the rapidly moving parts of the prominence (first 2 frames) at the WNW limb and also some bright "linear" structures (last 2 frames) falling back toward the surface at a slow rate.

microscopic process of a very similar erupting event observed with TRACE at even higher resolution is proposed by Filippov \& Koutchmy (2002). This last TRACE event did not produce a CME which would mean that sudden heating and filament eruption is not always followed by a CME.

\subsection{LASCO observations}

Figure 9 shows the result of the eruption further out in the W-L corona. Due to the masking effect by the occulting disk, it is not possible to get any data relevant to the start of the event. However, it is clear that the first component of the CME emerged from the diffraction rings of the occulting disk situated near 2.5 solar radii from the center, at 22:30 UT, as a bright edge at WNW. This is close to the position of the high arch described before, using the eclipse image taken in Iran. We suggest that they are indeed the same structure, the bright edge being the top part of the bright arch of the eclipse image.

\subsection{The high "arch" and the CME}

Figure 10 shows a partial frame of the eclipse image before processing. It reflects the distribution of intensities recorded on the film through the neutral density filter after removing the 


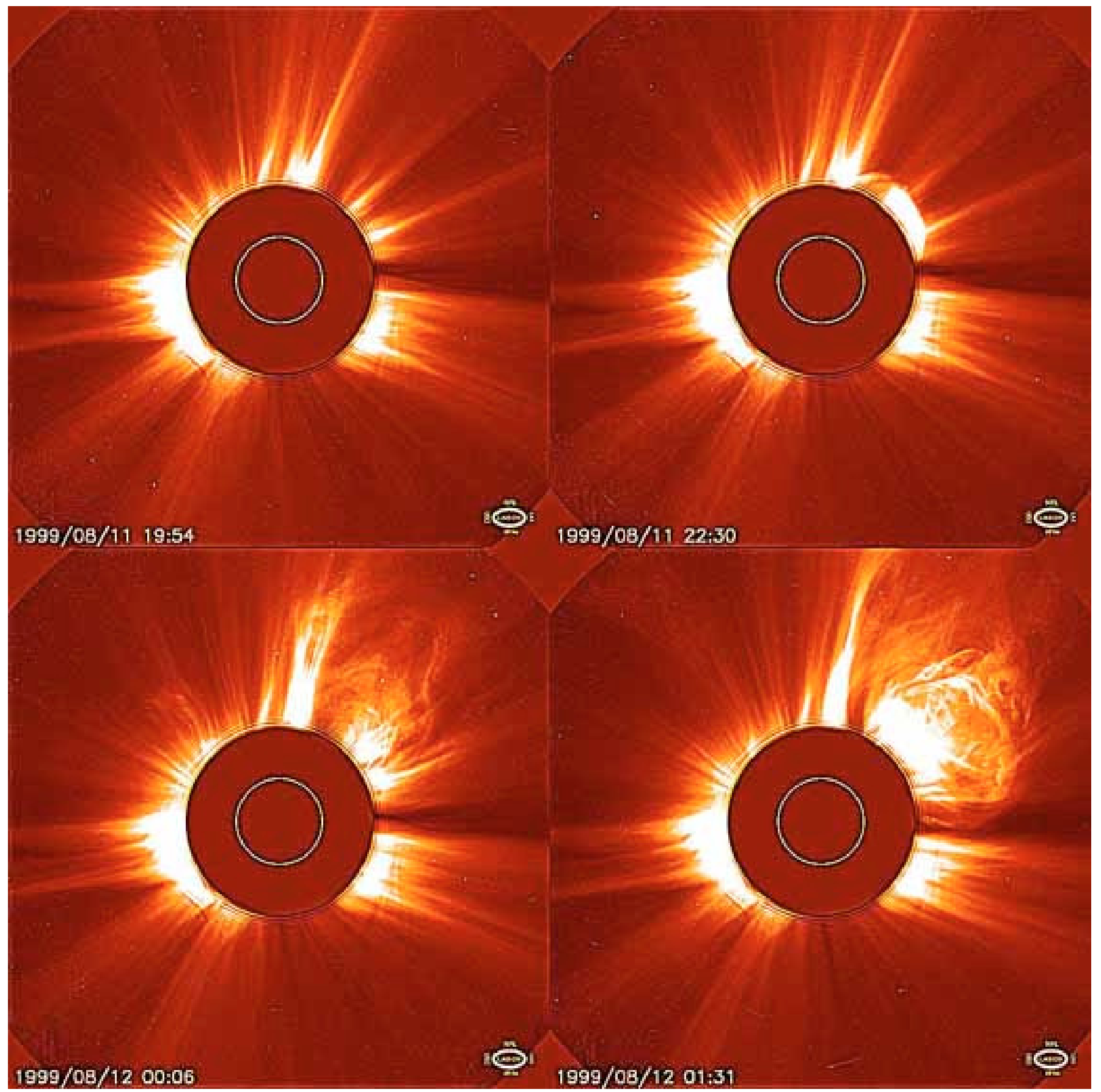

Fig. 9. A sequence of four W-L images taken with the Lasco-C2 coronagraph of SoHO, showing the CME event to the WNW. Further, the event was well recorded with the $\mathrm{C} 3$ coronagraph of larger field of view (not shown here). Note the very bright feature appearing at the edge of the occulter at WNW, at 22:30.

quasi-hydrostatic radial gradient. For the inner parts of the corona, we superposed, after properly scaling and centering, a single color image of very short exposure time taken when the limb of the Moon was very close to the photospheric limb. It is clear that the high bright arch does not show a shape that would be expected in the case of flux tubes and conservation of magnetic flux when field strength decreases with height. The top is narrower than the bottom, which is the opposite of what would have been seen with a flux tube anchored in the photosphere (because of the law of conservation of magnetic flux inside the tube). We do not know the 3D structure of the arch such that we cannot completely exclude the possibility that we see a dome in projection. In addition, the photometric measurements of the picture and their interpretation show that the mass of electrons contained in the top part of the arch can reproduce the bright edge that we see in Fig. 9 and is not very sensitive to the details of the 3D distribution. Comparison with a processed image obtained $1.5 \mathrm{~h}$ before in France by D. Fiel (from the "Société Astronomique de France") shows that the top of the arch was moving slowly outward with a velocity of the order 


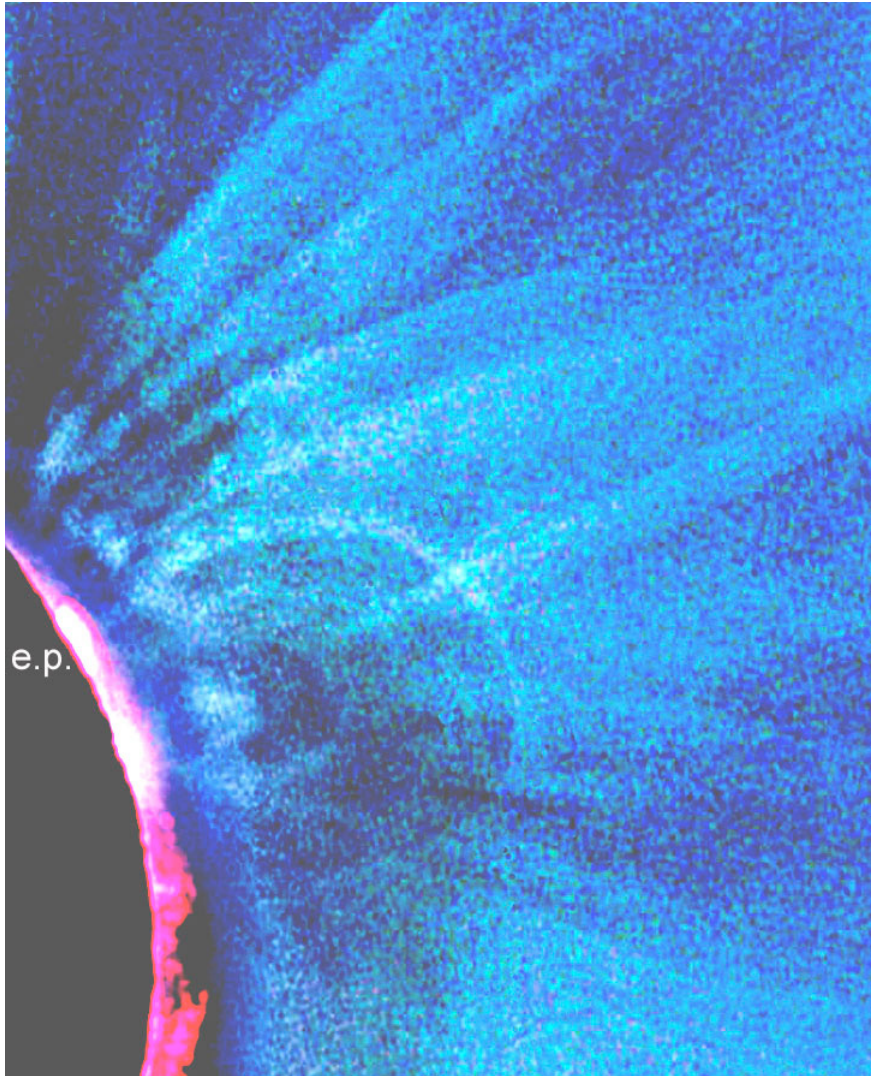

Fig. 10. A partial frame taken from the original eclipse image obtained at 12:03 UT through a radial filter, superposed on a single color image of the inner corona to better show the prominence emissions. The brighter the image, the denser the corona but the average radial gradient is removed: the effect of solar gravity is not visible although the whole field covers many scale heights. Note the high bright arch with a large cavity inside, the second smaller and weaker arch inside the large cavity with a smaller dark cavity below. The coronal enhancement is below the arch. The very bright prominence which later erupted is indicated by the sign "e.p.".

of $(3.5 \pm 2) \mathrm{km} \mathrm{s}^{-1}$. Many additional images were made later in Hungary and Turkey with different scales, which confirmed this slow motion. Finally, using the whole set of space-borne images, we measured the apparent projected positions of the CME components. The result shows an apparent velocity of $440 \mathrm{~km} \mathrm{~s}^{-1}$ (Fig. 11).

\section{Discussion and conclusions}

The average velocity deduced from Fig. 11 is very close to the average speed of all CMEs studied in the literature. It makes the studied case a prototype of a limb CME. The extrapolation towards the surface of the proper motion from LASCO C 2 and C3 images does not fit with the top part of the ejected prominence seen on EIT images. It suggests that the high arch could be at the origin of the first bright front of the CME, reminiscent result obtained from the analysis of SMM data (Harrison 2000). The high arch we saw to the WNW during the eclipse looks like a good candidate to be the precursor missing in all studies performed before in the absence of acceptable observations of the intermediate corona (near $1.6 R_{\odot}$ ). It is important

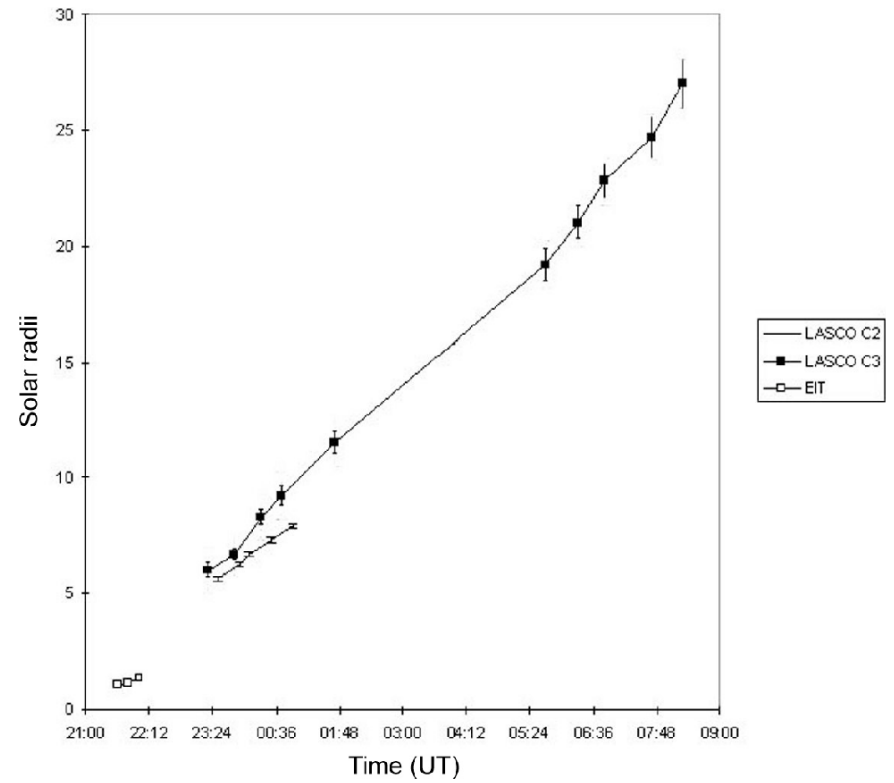

Fig. 11. The successive positions of selected parts of the CME from the Sun center, from EIT (top part of the prominence) and Lasco C2 and $\mathrm{C} 3$ images.

to understand the physics of the coronal cavities which could be the destabilizing agent for the whole region (see November \& Koutchmy 1996 for a discussion of the pressure equilibrium conditions). TRACE images in Fe XII show a strong coronal enhancement located immediately under the cavity "system" which reveals an active region there, possibly beyond the limb.

The large-scale eclipse image did not show a helmet streamer with cusp above the high arch. A naïve interpretation of what we see on the eclipse image (Fig. 10) would be to suggest a large magnetic "cloud" making a cavity supporting and/or pushing away the arch or the dome against the solar gravity, and that the structure erupted as a result of forces due to the buoyancy. Other forces can be at work, including magnetic forces (see Chen et al. 2000). The mechanism proposed in that paper starts with an erupting flux rope directly driven out of equilibrium by the increasing poloïdal magnetic flux on a timescale of hours. One difficulty comes from the unknown behaviour of a magnetic rope, visualised as the absence of plasma there. The filament eruption process, and the activity observed in the nearby coronal enhancement before, cannot be described by an increase of poloïdal flux alone, as the latter is not associated with heating. Reconnections are an other possibility, but we lack definite observational signature(s). The scenario recently advanced by Antiochos et al. (1999) looks very promising. The instability assumed by these last authors may result from shearing motion at the feet of the magnetic field lines which are forcing reconnections. Reconnections are produced under the pressure of buoyancy forces because, as noted here, the cavity extends over 5 to 10 pressure scales. Further, during the dynamical phase (see Figs. 8 and 9) evidence of structures flowing back toward the surface exists. However, we cannot conclude that the driving force is only due to the buoyancy (see Vourlidas et al. 2000). 


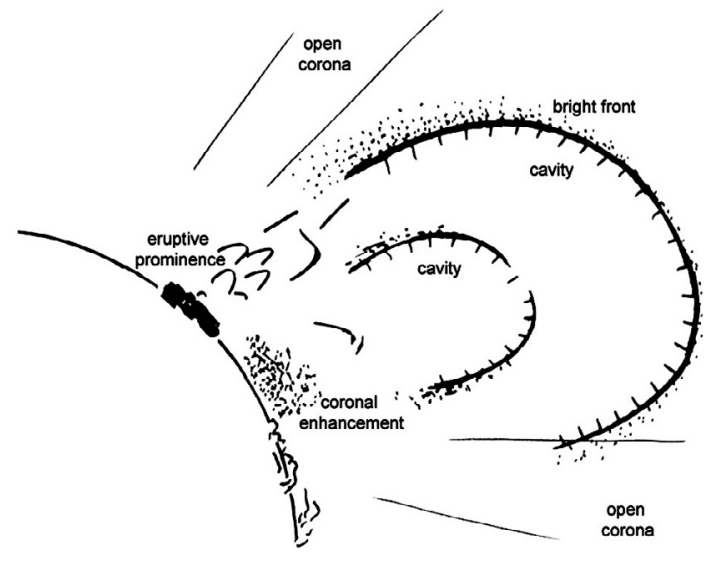

Fig. 12. Model to explain all important observations around the CME reported in this paper. The internal part is similar to model A of Fig. 1, although no helmet streamer is involved and the prominence is on the side. We added the "high cavity", which is slowly rising for several hours, due possibly to a buoyancy effect, producing a "bright front" by compression of the surrounding plasma.

Inside the shell defined by the high arch, the multiple magnetic cavities conductively "insulate" the inner parts and permit the heating in the coronal enhancement (waves, reconnections). The inserted prominence slowly rises as a result of the heating. Current loops are possibly produced in the inner corona. Ultimately, they have disrupted, producing the ejection and heating to coronal temperatures of the top part of the filament. Such an instability mechanism has been reported in modelling SXR jets (see Yokoyama \& Shibata 1996), but only in the 2D case, which excludes any "twisting" phenomenon related to the dynamical phase. The numerical simulations of these authors should now be extended to the $2.5 \mathrm{D}$ case (see Koutchmy \& Molodensky 1993). We observe the twist here in the 1999 eclipse CME, as well as in other cases of CMEs (including cases of a high latitude CME when the filament eruption was not identified, Boulade et al. 1997) in parts of the CME possibly connected to the surface, in the low corona (Airapetian \& Koutchmy 1994). It seems that the signature of twists is related to the intense and sudden heating of the ejected "cool" prominence plasma, an observation rarely noticed (see also the CME model of Filippov 1996). The question of heating during the CME process and, conversely, of the radiative output, and subsequent cooling of parts falling back, is beyond the scope of this paper. We however feel that it should be considered in the future, including the question of why the event seems to keep some parts of the erupting filament with the same but expanded morphology up to very long distances, implying a rather low detwisting effect.

Figure 12 is an attempt to visualize the early phase of the $\mathrm{CME}$, as shown in the interpretation of both eclipse results and spaceborne images, to propose areas in which the dynamics of the cavity would play an important role, including buoyancy. Unfortunately, we cannot explain the apparent link between the behavior of the high arch and the cavity system, the coronal enhancement below the arch and the erupting prominence to the side.

Acknowledgements. Eclipse observations were supported by the PNST of CNRS-France, by CNES-France and by Institut d'Astrophysique de Paris and they benefited from the help of many people on the site in Iran, including J. Mouette (IAP-CNRS France) and M. Laal Aaly (Tabriz Univ.). J. Mouette performed a large part of the processing of the 3 meter focal length image used in this paper. P.-A. Grorod, S. Borger, D. Fiel, D. Oropeza and Th. Sanchez from IAP and B. Filippov, M. Molodensky, I. Veselovsky from Moscow, A. Nikoghossian, from Burakan (Armenia), helped in evaluating the data. Space-borne observations are supported by both NASA and ESA. Analysis of TRACE data at the CfA is supported by a contract from Lockheed Martin. We thank J. Gurman and J. Klimchuk for their interest and F. Auchère, the staff of NRL, of CfA and of Lockheed present at GSFC during eclipse observations, for the help in programming, collecting and pre-processing the data. We acknowledge the very good work done by our referee in closely reviewing a first draft of the paper.

\section{References}

Adjabshirizadeh, A., Baudin, F., Bocchialini, K., et al. 1999, ESA SP448,1283

Airapetian, V. S., \& Koutchmy, S. 1994, ESA SP-373, 195

Allen, C. W. 2000, Astrophysical Quantities, 4th version, ed. A. N. Cox (AIP Press \& Springer), 359

Antiochos, S. K., DeVore, C. R., \& Klimchuk, J. A. 1999, ApJ, 510, 485

Boulade, S., Delannée, C., Koutchmy, S., et al. 1997, ESA SP-404, 217

Bocchialini, K., \& Koutchmy, S. 2001, in Proc. IAP Conf. 2000 on Total eclipses and Coronal Physics, ed. Durand, \& Koutchmy, SAF Pub., Obs. et Travaux, 53, 60

Chen, J., Santoro, R. A., Krall, J., et al. 2000, ApJ, 533, 481

Filippov, B. 1996, A\&A, 313, 277

Filippov, B., \& Koutchmy, S. 2002, Sol. Phys., 208, 283

Gibson, S. E., \& Low, B. C. 1998, ApJ, 493, 460

Harrison, R. A. 2000, in Encyclopedia of Astronomy and Astrophysics, 2498 (UK: Institute of Physics Publ.)

Koutchmy, S., \& Lamy, P. 1985, ed. Giese, \& Lamy (Riedel Pub.), Proc. IAU Coll., 85, 63

Koutchmy, S. 1992, ESA SP-348, 73

Koutchmy, S., \& Molodensky, M. 1993, Lect. Notes Phys., Adv. Sol. Phys., 432, 167

Koutchmy, S. 1994, Adv. Space Res., 14, 29

Koutchmy, S., \& Nikoghossian, A. 2002, A\&A, 395, 983

Krall, J., Chen, J., \& Santoro, R. 2000, ApJ, 539, 964

Luhmann, J. G., Li, Y., Zhao, X., \& Yashiro, S. 2003, Sol. Phys., 213, 367

Molodensky, M., Starkova, L., Koutchmy, S., \& Ershov, A. 1996, ASP Conf. Ser., 95, 385

November, L., \& Koutchmy, S. 1996, ApJ, 446, 512

Priest, E. R., \& Forbes, T. G. 2000, Magnetic Reconnection (Cambridge Univ. Press)

Vourlidas, A., Subramanian, P., Dere, K. P., \& Howard, R. A. 2000, ApJ, 534, 456

Yokoyama, T., \& Shibata, K. 1996, PASJ, 48, 353 